\title{
Maternal and Fetal Fatty Acid Profile in Normal and Intrauterine Growth Restriction Pregnancies With and Without Preeclampsia
}

\author{
GIOIA ALVINO, VERONICA COZZI, TATJANA RADAELLI, HENAR ORTEGA, EMILIO HERRERA, AND IRENE CETIN
}

Unit of Obstetrics and Gynecology, Department of Clinical Sciences "L. Sacco," University of Milan, Italy and Centre for Fetal Research Giorgio Pardi, University of Milan, Italy [G.A., V.C., I.C.]; Department of Mother, Child and Neonate "L. Mangiagalli,” IRCCS Ospedale Maggiore Policlinico Mangiagalli e Regina Elena, Milan, Italy [T.R.]; Department of Biochemistry, Molecular and Cell Biology,

University San Pablo-CEU, Madrid 28660, Spain [H.O., E.H.]

\begin{abstract}
The aim of this study was to evaluate maternal and fetal lipid profile in intrauterine growth restriction (IUGR) pregnancies with and without preeclampsia (PE). Thirteen normal pregnancies studied during the third trimester (control M) and 29 at elective cesarean section (control CS) were compared with 18 pregnancies complicated by IUGR (IUGR only) and with seven pregnancies complicated by both IUGR and PE (IUGR-PE). Total plasma fatty acids, triglycerides, cholesterol, and nonesterified fatty acids (NEFA) were determined in maternal and fetal plasma. Nutritional intake was analyzed. IUGR only mothers had lower percentage of linoleic acid (LA) and higher arachidonic acid (AA) than controls, partly explained by higher AA dietary intake. Higher levels of NEFA were observed both in IUGR only and in IUGR-PE mothers whereas triglyceride levels were increased in IUGR-PE mothers only. In IUGR-PE fetuses, LA and AA were significantly decreased, whereas triglyceride and NEFA concentrations were significantly increased compared with normal fetuses. In conclusion, IUGR only is associated with altered fatty acids profile not completely accounted by dietary changes. We hypothesize that the differences observed in IUGR with PE for triglycerides and other lipids could be related to a difference in maternal phenotype. (Pediatr Res 64: 615-620, 2008)
\end{abstract}

$I^{n}$ ntrauterine growth restriction (IUGR) is characterized by a deficiency of fetal growth and a failure of the conceptus to follow its growth potential. This condition is considered a severe disease because it can be associated with adverse outcomes during pregnancy, labor, and the neonatal period (1-4). Moreover, shifts during perinatal growth have pronounced effects on neonatal and adult health $(5,6)$. In recent years, a number of studies have reported a correlation among low birth weight and cardiovascular and metabolic diseases during adult age (7).

IUGR has been correlated to a specific placental phenotype based on the evidence that defective fetal nutrition is related to inadequate transport function (8). We have previously dem-

Received January 22, 2008; accepted July 12, 2008

Correspondence: Irene Cetin, M.D., Unit of Obstetrics and Gynecology, Department of Clinical Sciences "L. Sacco," University of Milan, Via G. B. Grassi, 74, 20151 Milano, Italy; e-mail: irene.cetin@unimi.it

Supported financially by the Commission of the European Communities, within the FP 5 Key Action 1: Food, Nutrition and Health (Perilip. QLK1-2001-00138 Influence of Dietary Fatty Acids on the Pathophysiology of Intrauterine Foetal Growth and Neonatal Development). It does not necessarily reflect the views of the Commission and in no way anticipates its future policy in this area. onstrated that placental supply of amino acids is reduced in IUGR fetuses independently from the severity of growth restriction and from the presence of hypoxia (9). Moreover, maternalfetal gradient of glucose are increased in severe IUGR fetuses, i.e., those with alterations of umbilical blood flow (10).

The dietary essential fatty acids, linoleic (18:2 n-6, LA), $\alpha$-linolenic (18:3 n-3, LNA), and their long chain polyunsaturated derivatives (LCPUFA), mainly arachidonic acid (20:4 n-6, AA) and docosahexaenoic acid (22:6 n-3, DHA), are important structural elements of cell membranes, which are needed for the normal development of the CNS and retina (11). We have previously reported that IUGR fetuses studied in utero at the time of fetal blood sampling are characterized by reduced conversion ratios of LCPUFA from their derivatives (12).

Preeclampsia (PE) represents another important cause of perinatal morbidity and mortality (13). The typical clinical manifestations of $\mathrm{PE}$, including hypertension, proteinuria, and the varying degrees of ischemic peripheral organ damage, which typically arise in the third trimester of gestation, might represent late phenomena of the complex process of implantation and placental formation (14).

Therefore, IUGR and PE might represent different aspects of placental insufficiency caused by a deficient remodeling of the spiral arteries during the interaction between maternal and fetal sides at the time of trophoblast invasion $(15,16)$.

Plasma concentrations of both triglyceride-rich lipoproteins and nonesterified fatty acids (NEFA) are significantly increased in women who develop PE relative to normal pregnant women (17), contributing to the endothelial dysfunction of PE by serving as substrates for lipid peroxidation with the generation of free radicals, lipid peroxides, and reactive oxygen species (17-19). On the other hand, an unbalanced ratio between tromboxane and prostacyclins, both derivatives of $\mathrm{AA}$, has been recognized as one of the steps leading to placental hypoxia in pregnancy-induced hypertension (20). Moreover, tromboxane and prostacyclins are also mediators of

\footnotetext{
Abbreviations: AA, arachidonic acid; AA/LA, arachidonic acid/linoleic acid ratio; CS, cesarean section; DHA, docosahexaenoic acid; DHA/LNA, docosahexaenoic acid $/ \alpha$-linolenic acid ratio; IUGR-PE, intrauterine growth restriction and preeclampsia; LA, linoleic acid; LCPUFA, long chain polyunsaturated fatty acids; LNA, $\alpha$-linolenic acid; NEFA, nonesterified fatty acids
} 
the inflammatory process that have recently been involved in the systemic impact of placental insufficiency (18-20).

In the present study, maternal and fetal total plasma fatty acids have been compared in normal and IUGR pregnancies evaluating the influence of maternal dietary intakes and the potential differences in presence of PE.

\section{METHODS}

The studies were performed in the Unit of Obstetrics and Gynecology of the DMCO San Paolo Hospital, Milan and in the Unit of Obstetrics and Gynecology of the Clinica Mangiagalli, Milan, in a period of 4 y (October 2000-June 2005). The University of Milan Ethical Board approved the protocol of the study. Informed consent was obtained from all pregnant women.

Clinical studies. Forty-two normal pregnancies (controls) and 25 pregnancies complicated by IUGR were studied. Gestational age was calculated from the last menstrual period and confirmed by routine ultrasonography at $20 \mathrm{wk}$ of gestation. Exclusion criteria were maternal diseases, gestational diabetes, alcohol abuse, drug addiction, abnormal fetal karyotype, and fetal malformations or infections. All pregnancies were singleton and none of the women smoked during pregnancy.

Controls were normal pregnancies that delivered appropriate for gestational age birth weight infants according to Italian birth weight-gestational age standards (21). Twenty-nine controls were studied at the time of elective cesarean section (control CS) performed because of previous CS, breech presentation, or maternal choice. Thirteen controls (control M) were sampled during the third trimester at an average gestational age (33.6 $\pm 2.1 \mathrm{wk})$ similar to the timing of delivery for the IUGR group. These pregnancies were followed until delivery to confirm normal birth weight at term and normal pregnancy outcome.

IUGR was identified in utero as a reduction in fetal growth rate by measurements of abdominal circumferences below the 10th percentile of reference values for fetuses of similar ages or by a decrease of more than 40 percentiles from the growth curve (22). Growth restriction was confirmed at birth if the neonatal weight was below the 10th percentile according to Italian standards for birth weight and gestational age (21). All pregnancies complicated by IUGR performed an elective CS in the interest of the fetus according to our clinical protocol.

Table 1 summarizes the characteristics of the population. As expected, IUGR only pregnancies were delivered at an average gestational age (34.1 \pm $3.0 \mathrm{wk}$ ) significantly lower than for normal pregnancies ( $38.6 \pm 0.5 \mathrm{wk})$. Therefore, IUGR only mothers were compared with controls of similar gestational age (control M) whereas IUGR only fetuses were compared with controls studied at CS (control CS). Birth weight and placental weight, as well as gestational age at delivery were significantly lower in IUGR only compared with both control groups.

Seven women with IUGR were also affected by preeclampsia (IUGR-PE) defined by the presence of hypertension and proteinuria (23).

Maternal venous blood samples were obtained in all pregnancies after overnight fasting. Time since last meal was always between 10 and $12 \mathrm{~h}$. In pregnancies studied at cesarean section (control CS and all IUGR), maternal venous blood samples were obtained simultaneously with umbilical arterial and venous blood samples collected from a doubly clamped segment of the cord immediately after fetal extraction.
Pregnant women were interviewed with a nutritional questionnaire to evaluate the characteristics of their diets and to calculate nutrient intakes. This is a semiquantitative validated questionnaire composed of pictures of most common Italian foods in different amounts (24). All pregnant women had to indicate the frequency of every dish consumed during the month before sampling. Data were entered in software designed for calculations of nutrient quantities.

Biochemical analyses. Blood samples were collected in EDTA tubes and kept on ice until centrifugation. Plasma for fatty acid analyses was separated by centrifugation at $3000 \mathrm{rpm}$ for $10 \mathrm{~min}$ at $4{ }^{\circ} \mathrm{C}$ and frozen at $-80^{\circ} \mathrm{C}$ until analysis.

Lipids were extracted from $0.20 \mathrm{~mL}$ of plasma in chloroform $/$ methanol (2:1) containing $10 \mathrm{mg} / \mathrm{L}$ of BHT (25). Fatty acids were trans-esterified with methanolic hydrochloride, and fatty acid-methyl esters were separated and analyzed on a Perkin Elmer gas chromatograph (Autosystem; Norwalk, CT) as previously reported (26). Total plasma fatty acids were expressed as percentage $(\mathrm{g} / 100 \mathrm{~g})$ of all detected fatty acids with a chain length of 12-24 carbon atoms in the sample. Plasma cholesterol, triglycerides, and free fatty acids (NEFA) were determined by commercial kits (Spinreact, Gerona, Spain) for cholesterol and triglycerides, and Wako Chemicals $(\mathrm{GmbH}$, Neuss, Germany) for NEFA.

Statistical analysis. Data are presented as mean \pm SD. The significance of the differences between fatty acids in IUGR only, IUGR-PE, and control groups was calculated with an unpaired $t$ test and with a two factorial unvaried ANOVA with Bonferroni posttest. Because NEFA did not follow a normal distribution, we performed a Kruskall Wallis test with Bonferroni posttest. They are represented as Median \pm inter quartile range. Differences between fetal and maternal samples in control CS were calculated with a paired $t$ test.

\section{RESULTS}

Nutritional questionnaire results. Nutritional information was collected from normal and pathological pregnancies and pooled results are presented in Table 2. Total daily caloric intake, as well as total amount of proteins, carbohydrates, or lipids were not significantly different among the groups. No significant differences were obtained among different types of lipids, except for the intake of AA, which was significantly higher in IUGR compared with control pregnancies.

Comparison between maternal and fetal fatty acids in normal pregnancies. Table 3 presents fatty acids in maternal and umbilical venous plasma of control pregnancies at term (control CS). The percentage of total saturated fatty acids was significantly higher in fetal than in maternal plasma, the difference corresponding to stearic acid (18:0) whereas palmitic acid (16:0) was lower in fetal than in maternal plasma. The proportion of oleic acid (18:1 n-9) was lower in fetal than in maternal plasma, the difference in PUFA corresponding to a lower proportion of LA (18:2 n-6) whereas both AA and DHA were higher in the fetal side. These differences

Table 1. Characteristics of the studied population

\begin{tabular}{|c|c|c|c|c|}
\hline & \multicolumn{2}{|c|}{ Controls $(n=42)$} & \multicolumn{2}{|c|}{ IUGR $(n=25)$} \\
\hline & Control CS $(n=29)$ & Control M $(n=13)$ & IUGR only $(n=18)$ & IUGR-PE $(n=7)$ \\
\hline Age (y) & $32.0 \pm 5.2$ & $31.6 \pm 4.3$ & $32.7 \pm 4.2$ & $34.3 \pm 4.7$ \\
\hline BMI pre-pregnancy $\left(\mathrm{kg} / \mathrm{m}^{2}\right)$ & $22.4 \pm 4.0$ & $20.8 \pm 2.0$ & $23.2 \pm 4.9$ & $24.6 \pm 4.9^{*}$ \\
\hline Sampling (wk) & $38.6 \pm 0.3$ & $33.6 \pm 2.1 \dagger$ & $34.6 \pm 2.4 \dagger$ & $32.1 \pm 3.3 * \dagger \neq$ \\
\hline Delivery (wk) & $38.6 \pm 0.3$ & $38.5 \pm 1.7$ & $34.6 \pm 2.4 * \dagger$ & $32.1 \pm 3.3^{* \dagger t}$ \\
\hline Gender $(\mathrm{M} / \mathrm{F})$ & $17 / 12$ & $4 / 9$ & $8 / 10$ & $4 / 3$ \\
\hline Neonatal weight $(\mathrm{g})$ & $3335.2 \pm 304.4$ & $3246.1 \pm 328.6$ & $1715.0 \pm 523.7^{* \dagger}$ & $1284.3 \pm 399.2 * \dagger$ \\
\hline Placental weight (g) & $519.4 \pm 108.0$ & $589.2 \pm 107.7$ & $252.2 \pm 78.0^{*} \dagger$ & $252.3 \pm 114.1^{* \dagger}$ \\
\hline
\end{tabular}

Data are expressed as mean \pm SD. $t$ test was used to determine differences between the groups: $* p<0.05 v s$ control M, †p<0.05 vs control CS, $¥ p<0.05$ vs IUGR only.

Control CS, physiologic pregnancies studied at cesarean section; control M, mothers with physiologic pregnancies studied during the third trimester of pregnancy; IUGR only, pregnancies complicated by intra uterine growth restriction; IUGR-PE, pregnancies complicated by both IUGR and preeclampsia. 
Table 2. Nutritional questionnaire results

\begin{tabular}{|c|c|c|}
\hline Intakes/day & $\begin{array}{l}\text { Controls } \\
(n=39)\end{array}$ & $\begin{array}{c}\text { IUGR } \\
(n=22)\end{array}$ \\
\hline kcal & $2175.1 \pm 608.3$ & $1818.6 \pm 638.2$ \\
\hline Proteins $(\mathrm{g})$ & $98.1 \pm 28.5(17.5 \%)$ & $87.0 \pm 24.7(17.6 \%)$ \\
\hline Carbohydrates (g) & $324.1 \pm 93.1(61.0 \%)$ & $274.9 \pm 112.3(60.4 \%)$ \\
\hline Lipids (g) & $60.9 \pm 21.8(21.5 \%)$ & $48.1 \pm 23.1(22.0 \%)$ \\
\hline Cholesterol (g) & $272.2 \pm 121.2$ & $228.9 \pm 84.2$ \\
\hline Saturated fatty acids (g) & $43.1 \pm 19.7$ & $31.6 \pm 16.3$ \\
\hline $\begin{array}{l}\text { Monounsaturated fatty } \\
\text { acids (g) }\end{array}$ & $16.7 \pm 7.9$ & $15.1 \pm 9.2$ \\
\hline Oleic acid (g) & $3.3 \pm 1.6$ & $2.5 \pm 1.5$ \\
\hline Linoleic acid (g) & $13.9 \pm 13.4$ & $7.6 \pm 4.2$ \\
\hline Linolenic acid (g) & $1.3 \pm 0.5$ & $1.1 \pm 0.6$ \\
\hline Arachidonic acid (g) & $0.08 \pm 0.06$ & $0.16 \pm 0.13^{*}$ \\
\hline $\begin{array}{l}\text { Eicosapentaenoic } \\
\text { acid }(\mathrm{g})\end{array}$ & $0.10 \pm 0.14$ & $0.11 \pm 0.13$ \\
\hline $\begin{array}{l}\text { Docosahexaenoic } \\
\text { acid }(\mathrm{g})\end{array}$ & $0.08 \pm 0.10$ & $0.04 \pm 0.02$ \\
\hline
\end{tabular}

Data are expressed as mean $\pm \mathrm{SD}$, for proteins, carbohydrates, and lipids, percentage of total caloric intake is indicated.

$* p<0.05$ IUGR $v s$ controls. are similar to those previously reported (27). Plasma concentrations of cholesterol, triglycerides, and NEFA were significantly higher in control mothers than in the fetal compartment (Table 4) with ratios of 5:1, 9:1, and 6:1, respectively. No significant differences were observed between umbilical vein and artery for these values (data not shown).

Comparison between IUGR and control mothers. Table 3 presents the percentage of total plasma fatty acids in the four groups studied. The comparison between mothers at $34 \mathrm{wk}$ (control M) and mothers at CS showed differences only for palmitic acid and the ratio between DHA and LNA that was lower and for steric acid that was higher in control $\mathrm{M}$ than in control CS.

Total plasma values of IUGR only mothers were compared with controls of similar gestational age (control M). No difference was found between the two groups for total saturated fatty acids although there were some differences within individual fatty acids. Palmitic acid (16:0) was higher in IUGR only than in controls whereas stearic acid (18:0) was lower in IUGR only than in control women.

Table 3. Total plasma fatty acids in maternal and umbilical venous plasma

\begin{tabular}{|c|c|c|c|c|}
\hline Fatty acids $\mathrm{g} / 100 \mathrm{~g}$ & Control CS $n=29$ & Control $\mathrm{M} n=13$ & IUGR only $n=18$ & IUGR-PE $n=7$ \\
\hline \multicolumn{5}{|l|}{ Saturated } \\
\hline m.v. & $37.0 \pm 2.7$ & $38.0 \pm 4.6$ & $37.2 \pm 2.4$ & $38.7 \pm 2.4$ \\
\hline u.v. & $50.5 \pm 5.4$ & & $46.0 \pm 7.0$ & $53.9 \pm 3.5^{*}$ \\
\hline \multicolumn{5}{|l|}{ Polyunsaturated } \\
\hline m.v. & $32.1 \pm 4.0$ & $33.3 \pm 4.1$ & $31.1 \pm 3.6$ & $28.5 \pm 3.0$ \\
\hline u.v. & $26.6 \pm 3.4$ & & $27.5 \pm 2.5$ & $24.4 \pm 1.7$ \\
\hline \multicolumn{5}{|l|}{ Palmitic acid } \\
\hline m.v. & $27.3 \pm 2.4$ & $23.5 \pm 3.4 \dagger$ & $28.8 \pm 2.5 \ddagger$ & $28.8 \pm 1.6 \ddagger$ \\
\hline u.v. & $25.2 \pm 5.6$ & & $28.0 \pm 3.6$ & $28.8 \pm 4.4$ \\
\hline \multicolumn{5}{|l|}{ Stearic acid } \\
\hline m.v. & $7.0 \pm 2.7$ & $9.5 \pm 2.8 \dagger$ & $6.3 \pm 2.2 \ddagger$ & $7.2 \pm 2.0$ \\
\hline u.v. & $20.1 \pm 10.5$ & & $14.7 \pm 7.9$ & $18.7 \pm 7.9$ \\
\hline \multicolumn{5}{|l|}{ Oleic acid } \\
\hline m.v. & $26.8 \pm 3.0$ & $25.4 \pm 2.7$ & $25.8 \pm 3.0$ & $27.3 \pm 1.5$ \\
\hline u.v. & $19.5 \pm 2.6$ & & $20.0 \pm 4.0$ & $17.3 \pm 3.3$ \\
\hline \multicolumn{5}{|l|}{ LA } \\
\hline m.v. & $22.5 \pm 4.2$ & $24.1 \pm 4.3$ & 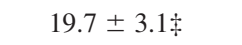 & $18.5 \pm 2.5 \neq$ \\
\hline u.v. & $7.9 \pm 1.6$ & & $8.6 \pm 1.7$ & $7.2 \pm 1.0$ \\
\hline \multicolumn{5}{|l|}{ LNA } \\
\hline m.v. & $0.4 \pm 0.3$ & $0.5 \pm 0.4$ & $0.2 \pm 0.1$ & $0.2 \pm 0.2$ \\
\hline u.v. & na & & na & na \\
\hline \multicolumn{5}{|l|}{$\mathrm{AA}$} \\
\hline m.v. & $5.0 \pm 0.9$ & $4.8 \pm 1.0$ & $5.8 \pm 1.1 \%$ & $5.1 \pm 0.9$ \\
\hline u.v. & $12.3 \pm 1.8$ & & $11.0 \pm 2.1$ & $10.3 \pm 2.4 \dagger$ \\
\hline \multicolumn{5}{|l|}{ EPA } \\
\hline m.v. & $0.6 \pm 0.6$ & $0.4 \pm 0.8$ & $0.5 \pm 0.4$ & $1.1 \pm 1.0$ \\
\hline u.v. & $1.2 \pm 2.4$ & & $0.9 \pm 1.4$ & $1.9 \pm 1.8$ \\
\hline \multicolumn{5}{|l|}{ DHA } \\
\hline m.v. & $2.4 \pm 0.5$ & $2.3 \pm 0.6$ & $2.2 \pm 0.5$ & $2.6 \pm 0.8$ \\
\hline u.v. & $4.0 \pm 0.9$ & & $3.8 \pm 0.6$ & $4.3 \pm 1.5$ \\
\hline \multicolumn{5}{|l|}{$\mathrm{AA} / \mathrm{LA}$} \\
\hline m.v. & $0.2 \pm 0.1$ & $0.2 \pm 0.1$ & $0.3 \pm 0.1 \dagger \ddagger$ & $0.3 \pm 0.0$ \\
\hline u.v. & $1.7 \pm 0.4$ & & $1.3 \pm 0.3 \dagger$ & $1.3 \pm 0.2$ \\
\hline \multicolumn{5}{|l|}{ DHA/LNA } \\
\hline m.v. & $6.8 \pm 2.6$ & $3.7 \pm 2.0 \dagger$ & $8.4 \pm 2.1 \dagger t$ & $10.5 \pm 7.4 \dagger \neq$ \\
\hline u.v. & na & & na & na \\
\hline
\end{tabular}

Data are expressed as mean \pm SD. Bonferroni's posttest was used to determine differences between the four groups after the ANOVA test: $* p<0.05 v s$ IUGR only, $\dagger p<0.05$ vs control CS, $\ddagger p<0.05$ vs control M.

u.v. and m.v. are always significantly different except when u.v. is reported in italics.

EPA, eicosapenahenoic acid; m.v., maternal vein; u.v., umbilical vein; na, not available. 
Table 4. Lipids in maternal and umbilical venous plasma

\begin{tabular}{|c|c|c|c|c|}
\hline & Control CS $(n=29)$ & Control M $(n=13)$ & IUGR only $(n=18)$ & IUGR-PE $(n=7)$ \\
\hline \multicolumn{5}{|c|}{ Cholesterol (mg/dL) } \\
\hline m.v. & $225.8 \pm 43.9$ & $243.5 \pm 47.2$ & $255.6 \pm 47.7$ & $267.9 \pm 43.0$ \\
\hline u.v. & $44.6 \pm 18.1$ & & $67.9 \pm 32.5$ & $59.7 \pm 31.0$ \\
\hline \multicolumn{5}{|c|}{ Triglycerides (mg/dL) } \\
\hline m.v. & $263.2 \pm 106.9$ & $208.6 \pm 54.2$ & $196.2 \pm 95.2$ & $316.4 \pm 95.5^{* \dagger}$ \\
\hline u.v. & $28.4 \pm 9.5$ & & $34.0 \pm 21.0$ & $49.4 \pm 13.9 \ddagger$ \\
\hline \multicolumn{5}{|l|}{ NEFA } \\
\hline m.v. & $\begin{array}{c}607.7 \\
(331.4-1027.0)\end{array}$ & $\begin{array}{c}319.0 \ddagger \\
(207.4-391.3)\end{array}$ & $\begin{array}{c}984.0 * \\
(624.2-1133.6)\end{array}$ & $\begin{array}{c}783.2 * \\
(691.6-1091.1)\end{array}$ \\
\hline u.v. & $\begin{array}{c}108.0 \\
(95.2-158.3)\end{array}$ & & $\begin{array}{c}205.5 \\
(110.3-275.4)\end{array}$ & $\begin{array}{c}240.2 \ddagger \\
(132.7-1014.8)\end{array}$ \\
\hline
\end{tabular}

Cholesterol and triglycerides are expressed as mean \pm SD. Bonferroni's posttest was used to determine differences between the four groups after the ANOVA test: $* p<0.05 v s$ control $\mathrm{M}, \ddagger p<0.05 v s$ control CS, $\dagger p<0.05 v s$ IUGR only.

NEFA are expressed as median (interquartile range). Bonferroni's posttest was used to determine differences between the four groups after the Kruskal-Wallis test. Different symbols in a row indicate differences $(p<0.05)$.

m.v., maternal vein; u.v., umbilical vein.

LA was significantly lower in IUGR only than in control pregnancies whereas AA was higher, whereas no significant differences were observed for 20:5 n-3 (EPA) or 22:6 n-3 (DHA).

The ratio between AA and LA (AA/LA) and between DHA and LNA (DHA/LNA) were significantly higher in IUGR only than in control mothers (Table 3).

As shown in Table 4, the concentration of NEFA in maternal plasma was significantly higher in IUGR only than in gestational age-matched controls (control $\mathrm{M}$ ) with no significant differences in the concentration of cholesterol or triglycerides.

Comparison between IUGR only and normal fetuses (control CS). Table 3 presents the comparison of umbilical vein total plasma fatty acids between IUGR only and control CS. No significant differences were found between the two groups for any of the fatty acids, except for the AA/LA ratio, which was significantly lower in IUGR than in control CS fetuses. The fetal-maternal ratio was significantly higher for the percentage of LA (control CS $=0.36 \pm 0.07$; IUGR only $=$ $0.43 \pm 0.10 ; p<0.01)$ and significantly lower for the percentage of AA (control CS $=2.49 \pm 0.46$; IUGR only $=$ $1.96 \pm 0.60 ; p<0.01)$ in IUGR only compared with normal pregnancies (data not shown). Comparison between umbilical and maternal plasma was similar to that observed in control CS pregnancies for all other fatty acids (Table 3 ). No differences were observed for cholesterol, triglycerides, and NEFA in umbilical venous plasma between IUGR only and controls (Table 4).

Differences in IUGR with PE. Table 3 shows the comparison between IUGR-PE and controls for maternal and fetal fatty acid profiles. No differences were found for any of the fatty acids between IUGR-PE and IUGR only in maternal or umbilical venous plasma, except for a higher proportion of saturated fatty acids in umbilical venous plasma in IUGR-PE than in IUGR only. Some differences were found in the comparison of maternal IUGR only and IUGR-PE plasma with controls. Palmitic acid was significantly higher both in IUGR only and IUGR-PE mothers than in their gestational age-matched controls (control M). In the n-6 fatty acid family, lower percentages of LA were found both in IUGR only and IUGR-PE maternal plasma versus control M, whereas AA and the ratio AA/LA were significantly higher only in IUGR only than in control mothers (control $\mathrm{M}$ ). In umbilical venous plasma, the percentage of AA was significantly lower than in controls (control CS) only in IUGR-PE, whereas the ratio AA/LA was significantly lower only in IUGR only than in controls. No difference between the IUGR and control groups in maternal or umbilical venous plasma was found for any of the fatty acids of the n-3 family (i.e., LNA or DHA) but DHA/LNA had a higher ratio in both IUGR only and IUGR-PE than in control mothers. NEFA concentrations were significantly higher in both IUGR-PE and IUGR only mothers than in controls (Table 4). In umbilical venous plasma, NEFA concentrations were significantly higher only in IUGR-PE versus controls. No significant difference was observed for cholesterol. Triglyceride concentrations were significantly higher in maternal plasma of IUGR-PE than in IUGR and in controls (Table 4) whereas in umbilical plasma, triglycerides were higher in IUGR-PE only versus controls (control CS).

Triglycerides, cholesterol, and NEFA had no relationship with maternal age or BMI when all data were pooled together or in the single groups of controls and IUGR (data not shown).

\section{DISCUSSION}

In the current study, we evaluated fatty acid profiles in mothers and fetuses of pregnancies complicated by intrauterine growth restriction. Furthermore, we evaluated whether the concomitant presence of PE was associated with a different maternal and/or fetal fatty acid phenotype.

In agreement with previous studies $(11,12,27,28)$, we reported higher percentages of LCPUFA (DHA and AA) and lower percentages of their essential precursors (LNA and LA) in fetal than in maternal plasma. This phenomenon has been called "biomagnification" and has been related to the capability of placenta and/or fetus to increase the percentages of LCPUFA in the fetal circulation, i.e., to provide the fetus with molecules that have been implied in CNS development in physiological conditions (11).

Evaluation of dietary intakes by nutritional questionnaires allowed to consider whether differences in plasma results were 
because of the differences in nutritional intakes between normal and IUGR pregnancies. Total caloric intakes, as well as intake of total proteins, carbohydrates, and lipids were similar in normal and IUGR mothers, except for a small, but significantly higher intake of AA in IUGR-complicated pregnancies compared with controls. Nutritional sources of AA are seed oils eggs, poultry, and pig meats (29). Although this nutritional difference could contribute to the higher proportion of AA found in maternal plasma of IUGR, its influence should be small because of its small proportion in the diet and the fact that the proportion of its synthetic precursor, LA, is much higher than that of AA in both the diet and plasma.

Despite the evidence of higher AA in mothers, the fetal fatty acid profile showed lower percentage of AA in fetal plasma of IUGR with PE. This difference might be related to inadequate placental function, similar to what was previously reported for other nutrients in IUGR (8-10). Possible mechanism responsible for the altered LC-PUFA profile could be changes in lipoprotein intake and degradation, and/or in fatty acids binding proteins $(30,31)$. A potential bias of these data is related to the different gestational age at the time of CS in IUGR compared with control pregnancies. However, we previously reported similar results in a smaller subset of IUGR pregnancies studied at fetal blood sampling, with a control group of comparable gestational age (12).

In the current study, we also report different fatty acid profiles in mothers of IUGR with or without PE. Mothers with IUGR only presented lower percentages of the n- 6 essential fatty acid LA associated with higher percentages of AA than controls of similar gestational age. This led to a higher ratio of AA/LA in IUGR only mothers. In pregnancies complicated by both IUGR and PE (IUGR-PE), AA was not significantly higher than in controls despite significantly lower percentage LA. Although this might be simply related to the failure to reach statistical power, another possible speculation is related to the increased arachidonic employment in the synthesis of molecules liable to the pathogenesis of PE, such as thromboxane (32). PE and IUGR are two pathological conditions involving an inflammatory process probably beginning in the placenta during the first stages of pregnancy (16). Several mediators of inflammation (prostaglandins, leucotrienes, and thromboxane) are derived from AA. From our data, we therefore hypothesize that increased availability of AA, (significant in IUGR; not significant in IUGR-PE) might lead to the production of inflammatory mediators (33).

We also observed a higher n-3 ratio of DHA/LNA both in IUGR and IUGR-PE mothers than in controls (control M) without any significant difference in the fetal compartment. This result is difficult to explain. The proportion of the fetal DHA is not significantly different in IUGR, but the level of LNA is very low and usually not detectable, therefore, it was not possible to calculate a conversion ratio for $n-3$ in the fetus.

Interestingly we also found significant differences in maternal plasma NEFA and triglycerides but not in cholesterol levels. Increased levels of NEFA are associated with increased maternal lipolysis in the last trimester of normal pregnancies $(27,34)$. We here report significantly higher NEFA levels in maternal plasma of IUGR only and in IUGR-PE mothers. This could be because of the increased adipose tissue lipolytic activity enhanced by the pathological stressing condition $(35,36)$ although decreased glycerol production has been recently reported in women carrying IUGR fetuses (37).

$\mathrm{PE}$ is a condition historically associated with hyperlipidemia and most of all hypertriglyceridemia (38) that is involved in the vascular damage typical of this syndrome (39): women with higher plasma lipid or triglyceride levels are at higher risk of hypertensive disorders and stroke due to atherosis (18) and in the same way they are exposed, in younger age, to higher risks of PE (38). Although cholesterol levels did not change, we observed higher levels of triglycerides in maternal plasma only of IUGR-PE and not of IUGR only. Differences in BMI should not account for this result, since no relationship was observed between triglycerides and BMI in our population. Previous studies have already reported increased levels of triglycerides in PE $(40,41)$ and triglycerides related vasculopathy and the inflammation cascade typical of PE (39) recognize the lipoperoxidation of fatty acids as one etiologic factor (42).

Increases of NEFA and triglycerides in the fetal circulation of only IUGR-PE could be the reflection of changes in placental lipase activities (31-43) in this pathological condition characterized by placental insufficiency but also by a vascular damage in which the atherogenic role of lipids and lipoperoxidation have been well established (18).

In summary, these results demonstrate that fatty acid profiles and plasma lipids are significantly altered in both maternal and fetal circulation of IUGR pregnancies, Moreover, a specific maternal and fetal fatty acid phenotype is demonstrated in IUGR pregnancies associated with PE. Because this is an observational study, the role of maternal, placental, and fetal factors in determining these differences needs to be further investigated.

Acknowledgments. We thank Milagros Morante for the excellent technical assistance and Edgardo Somigliana for his statistical support.

\section{REFERENCES}

1. Cetin I, Foidart JM, Miozzo M, Raun T, Jansson T, Tsatsaris V, Reik W, Cross J, Hauguel-de-Mouzon S, Illsley N, Kingdom J, Huppertz B 2004 Fetal growth restriction: a workshop report. Placenta 25:753-757

2. Visser GH, Sadovsky G, Nicolaides KH 1990 Antepartum heart rate patterns in small for gestational age third-trimester fetuses: correlations with blood gas values obtained at cordocentesis. Am J Obstet Gynecol 162:698-703

3. Pardi G, Cetin I, Marconi AM, Lanfranchi A, Bozzetti P, Ferrazzi E, Buscaglia M, Battaglia FC 1993 Diagnostic value of blood sampling in fetuses with growth retardation. N Engl J Med 328:692-696

4. Baschat AA 2004 Pathophysiology of fetal growth restriction: implications for diagnosis and surveillance. Obstet Gynecol Surv 59:617-627

5. Barker DJ 1990 The fetal and infant origins of adult disease. BMJ 301:1111

6. Barker DJ 1993 The intrauterine origins of cardiovascular disease. Acta Paediatr Suppl 82:93-99

7. Godfrey KM, Barker DJ 2001 Fetal programming and adult health. Public Health Nutr 4:611-624

8. Sibley CP, Turner MA, Cetin I, Ayuk P, Boyd CA, D'Souza SW, Glazier JD, Greenwood SL, Jansson T, Powell T 2005 Placental phenotypes of intrauterine growth. Pediatr Res 58:827-832

9. Cetin I, Ronzoni S, Marconi AM, Perugino G, Corbetta C, Battaglia FC, Pardi G 1996 Maternal concentrations and fetal-maternal concentration differences of plasma amino acids in normal and intrauterine growth-restricted pregnancies. Am J Obstet Gynecol 174:1575-1583

10. Marconi AM, Paolini C, Buscaglia M, Zerbe G, Battaglia FC, Pardi G 1996 The impact of gestational age and fetal growth on the maternal-fetal glucose concentration difference. Obstet Gynecol 87:937-942 
11. Crawford MA, Hassam AG, Williams G 1976 Essential fatty acids and fetal brain growth. Lancet 1:452-453

12. Cetin I, Giovannini N, Alvino G, Agostoni C, Riva E, Giovannini M, Pardi G 2002 Intrauterine growth restriction is associated with changes in polyunsaturated fatty acid fetal-maternal relationships. Pediatr Res 52:750-755

13. Sibai BM, Caritis S, Hauth J 2003 National Institute of Child Health and Human Development Maternal-Fetal Medicine Units Network. What we have learned about preeclampsia. Semin Perinatol 27:239-246

14. Meekins JW, Pijnenborg R, Hanssens M, McFadyen IR, van Asshe A 1994 A study of placental bed spiral arteries and trophoblast invasion in normal and severe pre-eclamptic pregnancies. Br J Obstet Gynaecol 101:669-674

15. Duckitt K, Harrington D 2005 Risk factors for pre-eclampsia at antenatal booking: systematic review of controlled studies. BMJ 330:565

16. Khong TY, De Wolf F, Robertson WB, Brosens I 1986 Inadequate maternal vascular response to placentation in pregnancies complicated by pre-eclampsia and by small-for-gestational age infants. Br J Obstet Gynaecol 93:1049-1059

17. Hubel CA, Roberts JM, Ferrel RE 1999 Association of pre-eclampsia with common coding sequence variations in the lipoprotein lipase gene. Clin Genet 56:289-296

18. Bayhan G, Kocygit Y, Atamer A, Atamer Y, Akkus Z 2005 Potential aterogenic roles of lipids, lipoprotein(a) and lipid peroxidation in preeclampsia. Gynecol Endocrinol 21:1-6

19. Raijmakers MT, Dechend R, Poston L 2004 Oxidative stress and preeclampsia. Hypertension 44:374-380

20. Granger JP, Alexander BT, Bennett WA, Khallil RA 2001 Pathophysiology of pregnancy induced hypertension. Am J Hypertens 14:178S-185S

21. Parazzini F, Cortinovis I, Botulus R, Fedele L 1991 [Standard of birth weight in Italy]. Ann Ostet Ginecol Med Perinat 112:203-246

22. Todros T, Ferrazzi E, Groli C, Nicolini U, Parodi L, Pavoni M, Zorzoli A, Zucca S 1987 Fitting growth curves to head and abdomen measurements of the fetus: a multicentric study. J Clin Ultrasound 15:95-105

23. ACOG Committee on Practice Bulletins-Obstetrics 2002 ACOG practice bulletin. Diagnosis and management of preeclampsia and eclampsia. Obstet Gynecol 99:159167

24. Porrini M, Gentile MG, Fidanza F 1995 Biochemical validation of a self administered semi-quantitative food frequency questionnaire. Br J Nutr 74:323-333

25. Folch J, Lees M, Stanley GH 1957 A simple method for the isolation and purification of total lipids from animal tissue. J Biol Chem 226:497-509

26. Amusquivar E, Rupérez FJ, Barbas C, Herrera E 2000 Low arachidonic acid rather than $\alpha$-tocopherol is responsible for the delayed postnatal development in offspring of rats fed fish oil instead of olive oil during pregnancy and lactation. J Nutr 130:2855-2865

27. Herrera E, Ortega H, Alvino G, Giovannini N, Amusquivar E, Cetin I 2004 Relationship between plasma fatty acid profile and antioxidant vitamins durino normal pregnancy. Eur J Clin Nutr 58:1231-1238
28. Ruyle M, Connor WE, Anderson GJ, Lowensohn R 1990 Placental transfer of essential fatty acids in humans: venous arterial difference for DHA in fetal umbilical eritrocytes. Proc Natl Acad Sci USA 87:7902-7906

29. Hibbeln JR, Nieminen LR, Blasbalg TL, Riggs JA, Lands WE 2006 Healthy intakes of n-3 and n-6 fatty acids: estimations considering worldwide diversity. Am J Clin Nutr 83:1483S-1493S

30. Tabano S, Alvino G, Antonazzo P, Grati FR, Miozzo M, Cetin I 2006 Placental LPL gene expression is increased in severe intrauterine growth-restricted pregnancies. Pediatr Res 59:250-253

31. Gauster M, Hiden U, Blaschitz A, Frank S, Lang U, Alvino G, Cetin I, Desoye G, Wadsack C 2007 Dysregulation of placental endothelial lipase and lipoprotein lipase in intrauterine growth-restricted pregnancies. J Clin Endocrinol Metab 92:22562263

32. Wetzka B, Nüsing R, Charnock-Jones DS, Schäfer W, Zahradnik HP, Smith SK 1997 Cyclooxygenase- 1 and -2 in human placenta and placental bed after normal and pre-eclamptic pregnancies. Hum Reprod 12:2313-2320

33. Bowen RS, Zhang Y, Gu Y, Lewis DF, Wang Y 2005 Increased phospholipase A2 and tromboxane but not prostacyclin production by placental trophoblast cells from normal and preeclamptic pregnancies cultured under hypoxia condition. Placenta 26:402-409

34. Diderholm B, Strisdberg M, Ewald U, Lindeberg-Norden S, Gustafsson J 2005 Increased lipolysis in non-obese pregnant woman studied in the third trimester. BJOG 112:713-718

35. Fischer T, Heusser K, Schobel HP 1999 The autonomic nervous system and preeclampsia. Zentralbl Gynakol 121:603-607

36. Lönnqvist F, Wennlund A, Wahrenberg H, Arner P 1992 Effect of mental stress on lypolysis in humans. Metabolism 41:622-630

37. Diderholm B, Stridsberg M, Norden-Lindeberg S, Gustafsson J 2006 Decreased maternal lipolysis in intrauterine growth restriction in the third trimester. BJOG 113:159-164

38. Ray JG, Diamond P, Singh G, Bell CM 2006 Brief overview of mathernal triglycerides as a risk factor for pre-eclampsia. BJOG 113:379-386

39. Ness RB, Sibai BM 2006 Shared and disparate components of the pathophysiologies of fetal growth restriction and preeclampsia. Am J Obstet Gynecol 195:40-49

40. Magnussen EB, Vatten LJ, Lund-Nilsen TI, Salvesen KA, Davey Smith G, Romunstad PR 2007 Prepregnancy cardiovascular risk factors as predictors of preeclampsia: population based cohort study. BMJ 335:978

41. Mayret-Mesquiti M, Pérez-Méndez O, Rodríguez ME, Fortoul TI, Gorocica P, Bernal-Alcántara D, Montaño LF, Alvarado-Vasquez N 2007 Hypertriglyceridemia is linked to reduce nitric oxide syntesis in women with hypertensive disorders of pregnancy. Hypertens Pregnancy 26:423-431

42. Qiu C, Phung TT, Vadachkoria S, Muy-Rivera M, Sanchez SE, Williams MA 2006 Oxidized low-density lipoprotein (oxidized LDL) and the risk of preeclampsia. Physiol Res 55:491-500

43. Innis SM 1991 Essential fatty acids in growth and development. Prog Lipid Res 30:39-103 